\title{
Discrete Sliding Mode Control Design for Piezoelectric Actuator
}

\author{
Van HUYNH-VAN $N^{1}$ and Phong TRAN-THANH ${ }^{2, *}$ \\ ${ }^{1}$ Modeling Evolutionary Algorithms Simulation and Artificial Intelligence, \\ Faculty of Electrical \& Electronics Engineering, Ton Duc Thang University, \\ Ho Chi Minh City, Viet Nam \\ ${ }^{2}$ Faculty of Electrical and Electronic Engineering, School of Science and Technology, \\ RMIT University, Ho Chi Minh City, Viet Nam \\ *Corresponding Author: Phong Tran-Thanh (Email: ttphong.auto.ltt@gmail.com) \\ (Received: 8-Aug-2019; accepted: 29-Sep-2019; published: 30-Sep-2019) \\ DOI: http://dx.doi.org/10.25073/jaec.201933.254
}

\begin{abstract}
Piezoelectric bimorph actuators have been employed in several applications. In this paper, the piezoelectric actuator is discretized and its hysteresis function is studied, then a digital sliding mode controller is designed. Furthermore, a perturbation estimation technique is applied and an observer is no longer needed. In addition, simulations are performed also using the traditional PID controller in order to validate the proposed controller scheme performance.
\end{abstract}

\section{Keywords}

\section{Discrete Sliding Mode Control, Hystere- sis, Piezoelectric, Micromanipulation}

\section{Introduction}

Piezoelectric actuators have become popular due to its capability to perform an extreme small displacement, ranging from microns to nanometer. This is an important characteristic, since many devices and applications require sub-micron or nanometer resolution, such as, microscopes [1][6], positioning stages [7]- [9], microgrippers [10][14]. However, due to the ferroelectric properties of the piezoelectric materials, these actu- ators show hysteresis behavior when a voltage is applied [15]. Since hysteresis is a nonlinear phenomenon, this causes inaccuracy and unsatisfactory tracking performance when a high precision is required. Therefore, development of a controller that can suppress nonlinear behavior is essential.

A piezoelectric actuator model can be described as an electromechanical system, where the electrical system is composed of nonlinear and linear functions, and mechanical system is a mass-spring-damper system. However, a control scheme is not given in [16]. Several authors propose a hysteresis model, for example, Preisach model [17], Maxwell model [18], Prandtl-Ishlinskii model [19], Bouc-Wen model [20], and Duhem model [21]. In these studies, the hysteresis model has an ellipsoidal shape or trapezoidal shape. In addition, in [22] the hysteresis function is described as a fourdimensional chaotic system and its circuit implementation is designed in [23].

In order to attenuate hysteresis, charge amplifiers were designed. However a control scheme is still needed [16]. Development of control system for piezoelectric actuators has attracted attention due to their dynamic complexity. Recently, different kinds of control schemes were proposed, such as, backstepping control [14], adaptive con- 
trol [24,25], robust control [26], iterative learning control [27], or combinations between these control schemes. Sliding mode controller has several advantages such as, simplified control scheme, external disturbance rejection and a low sensitivity to plant parameter uncertainty [28]. For implementation of sliding mode control, full state feedback is required. However in most of cases we have only the position data. So, a development of state observer is indispensable.

To deploy SMC on a sampled data system, discrete-time SMC (DSMC) is more interesting and attractive [31]- [33]. In general, DSMC can be classified into state-based and output-based approach. The development of former based on system status or status error [33]- [35], meanwhile this is done on the basic system or an output error system [36,37]. Generally, the process of putting a plan into both methods requiring feedback of system state. However, in the greater number of practical situations, the location information of a piezoelectric actuator system gave that displacement sensor. Therefore, a state observer is indispensable to implement the practical DSMC [33], [35]- [37], in which complicates the control design procedures. Moreover, the cause of instability of system is lack of stability for designed observer state. In this case, it is desirable to eliminate clearly the use of the state observer. However, the works are limited to make toward this issue. In the document of in the previous work, an input-output built an adaptive DSMC has been proposed [38,39], based solely on input and output data.

Ultimately, the motivation for this research is the development of a simple DSMC diagram without using complex hysteresis model and observing the state for precise motion control of a piezoelectric actuator. Specifically, unadjusted nonlinear effects are considered gross disturbances and the perturbation is estimated by using a one-step delayed estimation technique. Moreover, the avoidance of state observer is realized by developing a new DSMC based on the system with discrete-time second-order dynamics model. Local stability of closed loop system is proved on the theory and the effectiveness of the proposal scheme is confirmed through experimental test. According to the author's understanding, the proposed project is the simplest approach based on DSMC to carry out exclusively for piezoelectric actuator control $[15,16,30]$.

This paper focuses on to apply a control scheme for a trajectory tracking of a piezoelectric bimorph actuator. In section II, the electromechanical system is discretized with a fixed time step and hysteresis function is studied. Then in section III, a perturbation estimation technique is applied to estimate external disturbances and hysteresis. After finishing the previous steps, a discrete sliding mode controller is proposed and applied. In section IV, a PID controller is also designed and compared with the proposed discrete sliding mode controller. Finally, in section $\mathrm{V}$, we have the conclusion of this paper.

\section{DYNAMICS AND HYSTERESIS MODEL}

\subsection{Plant Model}

The piezoelectric bimorph actuator studied in this paper is illustrated in Fig. 1.

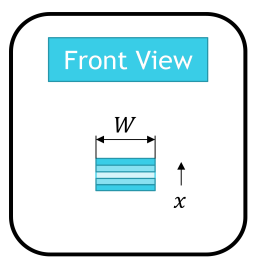

(a)

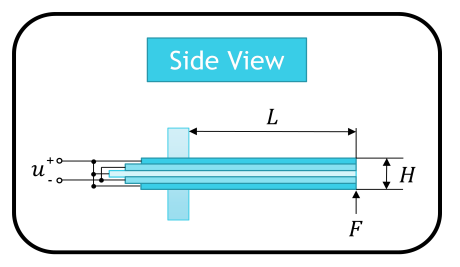

(b)
Fig. 1: Piezoelectric bimorph actuator. (a) front view (b) side view

When a voltage $\mathrm{u}$ is applied to a piezoelectric actuator, a force $\mathrm{F}$ is generated and the resultant displacement is given by $x$. The equation that describes the motion of a piezoelectric bimorph actuator is described as $[29,30]$

$$
M \ddot{x}(t)+B \dot{x}(t)+K x(t)=D u(t)+P(t)
$$

where $t$ represents the time variable, parameters $M, B$ and $K$, and $x$ are the mass, damping coefficient, stiffness, and displacement of the actuator, respectively. $D$ is the piezoelectric coefficient and $u$ is the input voltage. 
Remark 1. To be more information, Fig. 2. describes the overall electromechanical model of piezoelectric actuator. An equally accurate model was chosen for the piezo-phase due to be simply of implementation and accuracy to estimating the actual behavior of these actuators. The piezo-stage includes in a piezo-drive with a bending guided structure designed to have zero friction and friction. Additionally, the bending stages exhibit high rigidity or stiffness, high load capacity and insensitiveness to shock and vibration $[15,16]$.

The effects of hysteresis separated piezoelectric effects. $H$ shows the hysteresis effect and the voltage is $u_{h}$ due to this effect. The piezoelectric effect is regarded as $T_{\mathrm{em}}$ is an electromechanical converter with transformer proportion. Capacitance $\mathrm{C}$ points out the total power of individual piezoelectric wafers that are electrically in parallel to each other. Furthermore, $\dot{q}$ represents the total current flowing through the circuit. Specially, $q$ can be the total charge in the piezoelectric actuator. The charge $q_{p}$ presents the probe charge from the mechanical side. The voltage $u_{p}$ is due to piezo effect. The total voltage on the piezoelectric actuator is $u_{\text {peo }}, F_{p}$ is the force converted from the electrical side. The elongation of the piezoelectric actuator is denoted as $x=\Delta L$. The mechanical relationship between $F_{p}$ and $x$ is indicated by $m$. Note that we have equal electrical and mechanical energy at the interaction gates, i.e. $u_{p} q_{p}=F_{p} x[15]$.

In electromechanical model of piezoelectric actuator, $P(t)$ is the perturbation term, including hysteresis effect, external disturbances, and noise. When the input voltage $u=0$, no hysteresis will affect the piezoelectric actuator. In order to discretize the previous equation, both side are divided by $M$

$$
m \ddot{x}(t)+b \dot{x}(t)+k x(t)=d u(t)+p(t)
$$

where $m=1, b=B / m, k=K / M, d=D / M$, and $p(t)=P(t) / M$. For discretization, the Euler backward difference is adopted

$$
\begin{gathered}
\dot{x}(t)=\frac{1}{T}[x(k T)-x(k T-T)] \\
\ddot{x}(t)=\frac{1}{T^{2}}[x(k T)-2 x(k T-T)+x(k T-2 T)]
\end{gathered}
$$

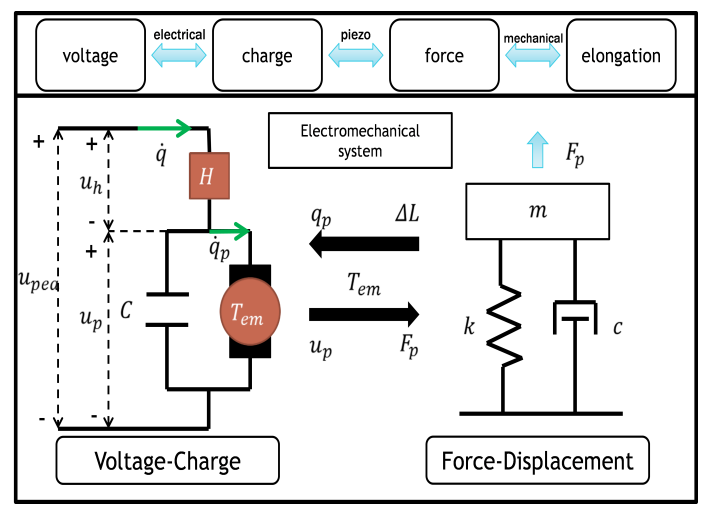

Fig. 2: Electromechanical representation of the piezoelectric actuator.

where $k$ represents the $k$ th time step. Therefore, substituting the equations (3), (4) into equation (2), we have the equivalent discrete time dynamics equation [30]

$$
\begin{aligned}
\bar{m} x(k T-2 T) & +\bar{b} x(k T-T)+\bar{k} x(k T) \\
& =\bar{d} u(k T)-p(k T)
\end{aligned}
$$

where $\bar{m}=1 / T, \bar{b}=(-b / T)-(2 / T), \bar{k}=k+$ $(b / T)+\left(1 / T^{2}\right)$, and $\bar{d}=d$.

Remark 2. To get more accuracy in the hysteresis model, the classic Preisach model was modified by adding a parallel term as shown in Fig. 3. The new term with hysteresis function has some effects as following [18]- [20], [40,41]:

- To fill the predicted hysteresis loop to reduce the errors with the measured loop;

- To pose the splitting phenomenon between minor and major loops.

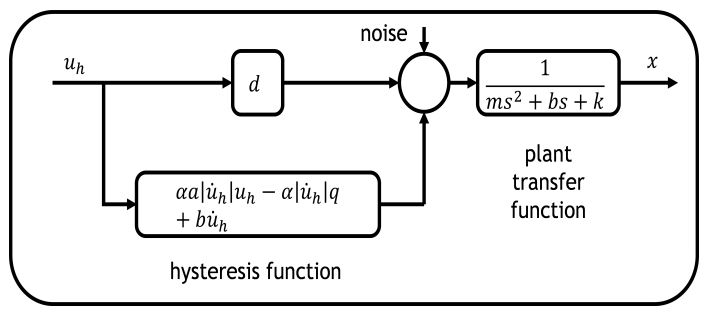

Fig. 3: Mathematical model of piezoelectric bimorph actuator in continuous time.

The purposed approach of this project is to continue the modeling of hysteresis topic. It 
also discusses and finds out the development of the modified Preisach model to replace the classic Preisach model. Therefore, the new model can be used to describe the nonlinear hysteresis loop of piezoelectric bimorph actuator under arbitrary or stimulating non-periodic input [18][20], [40,41].

Proposed Preisach model can be modified to upgrade the mathematical model of piezoelectric bimorph actuator to control the system.

The definition of the hysteresis effect is dynamic, nonlinear system and independent of ratio. So, we mean independent of the time scale in terms of rate-independence. In [40,41], the effect of a combination of basic elements is modeled. Therefore, the great number of parameters is relatively large in this model. Moreover, a model is not appropriate to proposed controller design. Consequently, in [42], a differential equation with only three parameters is used as application as a hysteresis model and a differential equation is also more appealing when we want to apply the model as a basis scheme for controller design.

In this part, we consider this equation in more detail. However, in [42], a much broad discussion can be search.

The hysteresis loop is a definition as a static loop in the input / output plane for a semistatic monotonic oscillating input such as low sinusoidal frequency.

The equation is proposed in [43] that considers first-order differential equation. It was developed to describe the magnetic hysteresis, but in [42] it has been experimentally verified that this differential equation is also suitable to describe the electrical delay as in the piezoelectric actuator. The model for the hysteresis effect between $u_{h}$ and $q$ is given by [15]:

$$
\dot{q}=\alpha\left|\dot{u}_{h}(t)\right|\left(f\left(u_{h}(t)\right)-q\right)+\dot{u}_{h}(t) g\left(\dot{u}_{h}(t)\right)
$$

where $f\left(u_{h}(t)\right)$ and $g\left(u_{h}(t)\right)$ are functions with which you can "shape" the hysteresis loop.

Therefore, we can have:

$$
f\left(u_{h}(t)\right)=a u_{h}(t), \quad g\left(u_{h}(t)\right)=b
$$

Where $a$ and $b$ are constants. So, the equation becomes:

$$
\dot{q}=\alpha a\left|\dot{u}_{h}(t)\right| u_{h}(t)-\alpha\left|\dot{u}_{h}(t)\right| q(t)+b \dot{u}_{h}(t)
$$

The hysteresis function shows in mathematical model of piezoelectric bimorph actuator in continuous time in Fig. 3.

\subsection{Perturbation Estimation}

In this paper, the model of continuous-time piezoelectric actuators is eliminated by applying the small sampling time $T$. Several approaches are available (e.g. zero-order hold) to get the purpose of discretization. In this study, Euler's backward difference is used due to its simplicity. For discretization, Euler's backward differences applied. A disturbance estimation technique developed by Elmali [29] was applied. A perturbation estimation technique developed by Elmali [29] is used.

It is necessary note that the backward differences (3) and (4) are used to discretize the continuous time model (2). We are not considered to discretize the noises at this project. The discrete-time model (5) exists in the delayed versions $x(k T-2 T)$ and $x(k T-T)$ of the plan of output $x(k T)$. It is nearly similar to other ways such as zero-order hold [30].

The main problem is discretized in the generated time delay $\approx T / 2$. The main reason of phenomenon of slow response in transient behavior of the closed loop control system is the time delay. In this study, a small sampling time $T$ will be selected when the time delay is ignored [15].

Therefore, the perturbation term $p(k T)$ is generated by its one-step delayed estimation based on the perturbation estimation technique.

$$
\begin{aligned}
& \hat{p}(k T)=p(k T-T)=-\bar{d} u(k T-T) \\
& +\bar{m} x(k T-3 T)+\bar{b} x(k T-2 T)+\bar{k} x(k T-T)
\end{aligned}
$$

if $\tilde{p}(k T)=\hat{p}(k T)-p(k T)$ is the perturbation estimation error, the equation (5) can be written as

$$
\begin{aligned}
\bar{m} x(k T-2 T) & +\bar{b} x(k T-T)+\bar{k} x(k T) \\
& =\bar{d} u(k T)+\hat{p}(k T)-\tilde{p}(k T)
\end{aligned}
$$


where $k$ represents the $k$ th time step. Perturbation estimation error depends on the sampling speed of the control hardware and the accuracy of the feedback sensing. Upper bound of this error can be experimentally determined.

The perturbation estimation error can be further expressed as:

$$
\begin{aligned}
\tilde{p}(k T) & =\hat{p}(k T)-p(k T) \\
& \approx-\dot{p} T=-\frac{T}{M} \dot{P}(t)
\end{aligned}
$$

\subsection{Hysteresis Effect}

The hysteresis effect is represented inside the electromechanical model as shown in Fig. 3 and can be described as a dynamic, rateindependent, and nonlinear [15]. In [16], a differential equation with three parameters and two variables is adopted to model the hysteresis effect. This equation and its discrete form are given by

$$
\dot{q}(t)=\alpha a\left|\dot{u}_{h}(t)\right| u_{h}(t)-\alpha\left|\dot{u}_{h}(t)\right| q(t)+b \dot{u}_{h}(t)
$$

$$
\begin{aligned}
& q(k T)=\left(\frac{1}{\frac{1}{T}+\alpha\left|\frac{1}{T}\left(u_{h}(k T)-u_{h}(k T-T)\right)\right|}\right) \\
& +\alpha a u_{h}(k T)\left|\frac{1}{T}\left(u_{h}(k T)-u_{h}(k T-T)\right)\right| \\
& +\frac{b}{T}\left(u_{h}(k T)-u_{h}(k T-T)\right)+\frac{q(k T-T)}{T}
\end{aligned}
$$

where $\alpha, a$ and $b$ are parameters.

In [15], it represents the method to find parameters by realistic hysteresis loop. Based on the experiment, it can find out $a$ and $b$ from center points and average slopes, the parameter $\alpha$ can then be experimentally determined from hysteresis areas. By varying these parameters, different shapes of hysteresis can be obtained in Fig. 4.

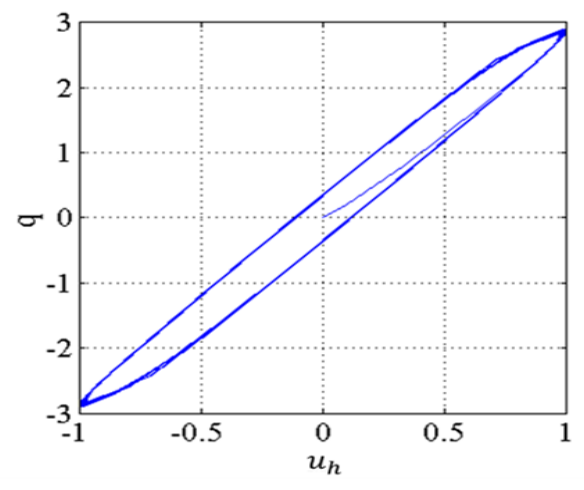

Fig. 4: Simulated hysteresis function.

\section{DIGITAL SLIDING MODE CONTROL}

Substituting the equation (9) into the discrete plant model (10), we have

$$
x(k T)=\frac{1}{k}\left[\begin{array}{c}
\bar{d}(u(k T)-u(k T-T)) \\
+\bar{m} x(k T-3 T) \\
(\bar{b}-\bar{m}) x(k T-2 T) \\
+(\bar{k}-\bar{b}) x(k T-T) \\
-\tilde{p}(k T)
\end{array}\right]
$$

The position error is defined as $e(k T)=x(k T)-$ $x_{d}(k T)$, where $x_{d}(k T)$ is the desired trajectory. A proportional-integral sliding surface is defined as,

$$
s(k T)=\lambda_{P} e(k T)+\lambda_{I} \varepsilon(k T)
$$

where $\varepsilon(k T)=e(k T)+\varepsilon(k T-T)$ is the integral error, $\lambda_{P}$ and $\lambda_{I}$ are proportional and integral gains. In this paper, the following reaching law is adopted,

$$
s(k T)-s(k T-T)=0
$$

Considering that the equivalent control $u(k T)$ is the solution to (19), the following deductions hold:

$$
\begin{gathered}
s(k T-T)=\lambda_{P} e(k T)+\lambda_{I} \varepsilon(k T) \\
s(k T-T)=\left(\lambda_{I}+\lambda_{P}\right) e(k T)+\lambda_{I} \varepsilon(k T-T)
\end{gathered}
$$

Then, inserting (14) into equation (17), and ignore the estimation errors $\tilde{p}(k T)$, leads to the 
equivalent control.

$$
\begin{aligned}
u(k T)= & u(k T-T) \\
+ & \frac{1}{d}\left[\begin{array}{l}
\frac{\bar{k}}{\lambda_{A}} s(k T-T) \\
\bar{k} \\
-\frac{\lambda_{A}}{\lambda_{I}} \lambda_{I}(k T-T) \\
+\bar{k} x_{d}(k T)
\end{array}\right] \\
- & \frac{1}{d}\left[\begin{array}{l}
\bar{m} x(k T-3 T) \\
+(\bar{b}-\bar{m}) x(k T-2 T) \\
+(\bar{k}-\bar{b}) x(k T-T)
\end{array}\right]
\end{aligned}
$$

where $\lambda_{A}=\lambda_{P}+\lambda_{I}$.

The equivalent control (19) represents the control action for the case of perfect disturbance estimation, that is, $\tilde{p}(k T)=0$. It takes effect in the sliding phase when the position trajectory is kept on the sliding surface $(s(k T)=0)$. However, if a large error $\tilde{p}(k T)$ occurs during the sliding phase, the standalone equivalent control cannot drive the position towards the sliding surface.

Thus, the equivalent control is augmented by a switching control $u_{S W}(k T)$ to give the total control action

$$
u(k T)=u^{e q}(k T)+u_{S W}(k T)
$$

Control input is given by

$$
\begin{aligned}
u(k T)= & u(k T-T) \\
+ & \frac{1}{d}\left[\begin{array}{l}
\frac{\bar{k}}{\lambda_{A}} s(k T-T) \\
\overline{\bar{k}} \\
-\frac{\lambda_{A}}{\lambda_{I} \varepsilon}(k T-T) \\
+\bar{k} x_{d}(k T)
\end{array}\right] \\
- & \frac{1}{d}\left[\begin{array}{l}
\bar{m} x(k T-3 T) \\
+(\bar{b}-\bar{m}) x(k T-2 T) \\
+(\bar{k}-\bar{b}) x(k T-T)
\end{array}\right] \\
- & \frac{\lambda_{s}}{\bar{d}} \operatorname{sgn}(s(k T-T)) .
\end{aligned}
$$

where $\lambda_{s}$ is a constant control gain and $\operatorname{sgn}(\cdot)$ represents the sign function. If $\lambda_{s} \geq|\tilde{p}(k T)|$, then the DSMC will occur with a quasi-sliding domain (QSD). The proof can found in [30]. However, when the actuator is operated under higher frequencies, it can decrease system performance. In electrical circuits, chattering phenomena can cause heating losses, degrading electronics components. In mechanical system, it may lead to high wears of moving mechanical parts. In this paper, a saturation function is adopted in order to attenuate the chattering effect. The saturation function is given by,

$$
\operatorname{sat}(k T)=\left\{\begin{array}{l}
\operatorname{sgn}(s(k T)) \text { if }|s(k T)|>\varepsilon \\
s(k T) / \varepsilon \text { if }|s(k T)| \leqslant \varepsilon
\end{array}\right.
$$

Remark 3. The chattering phenomenon is highly undesirable because it may excite high frequency unmodelled plant dynamics. There are some approaches to reduce the chattering. The first, the discontinuous function $\frac{s(k T)}{\|s(k T)\|}$ in the control input (21) is replaced by a continuous approximation such as $\operatorname{sat}(k T)$. This method cannot guarantee asymptotic stability but ultimate boundedness of system trajectories to within a neighborhood of the origin.

\section{SIMULATION RESULTS}

The plant parameters are shown in Table 1 . In this paper, a sampling time $T$ is chosen as $0.004 \mathrm{~s}$. So, the discrete plant parameters (5) are calculate using this value. In addition, for comparative study, the traditional PID is also implemented. The control input is given by,

$$
\begin{aligned}
u_{P I D}(k) & =u_{P I D}(k-1)+K_{p} e_{p}(k) \\
& +K_{i} e_{i}(k)+K_{d} e_{d}(k)
\end{aligned}
$$

where $u_{P I D}(k-1)$ is the control input in the previous time step, $K_{p}, K_{i}$, and $K_{d}$ are proportional, integral, derivative gains, respectively, and the proportional, integral and derivative errors are given by,

$$
\begin{aligned}
& e_{p}(k)=e(k)-e(k-1) \\
& e_{i}(k)=e(k) \\
& e_{d}(k)=e(k)-2 e(k-1)+e(k-2)
\end{aligned}
$$

Besides tracking performance analysis, we also calculate the percent maximum error (MAXE) 
Table 1. Parameters of the plant

\begin{tabular}{|c|c|c|}
\hline Symbol & Parameter & Value \\
\hline $\mathrm{M}$ & mass & $0.015 \mathrm{Kg}$ \\
\hline $\mathrm{B}$ & $\begin{array}{c}\text { damping } \\
\text { coefficient }\end{array}$ & $1 \mathrm{Ns} / \mathrm{m}$ \\
\hline $\mathrm{K}$ & stiffness & $1.5 \times 10^{6} \mathrm{Nm}$ \\
\hline $\mathrm{D}$ & $\begin{array}{c}\text { Piezoelectric } \\
\text { coefficient }\end{array}$ & $1.152 \mathrm{~N} / \mathrm{V}$ \\
\hline
\end{tabular}

Table 2. Parameters of the PID controller

\begin{tabular}{|c|c|c|}
\hline Symbol & Parameter & Value \\
\hline$K_{p}$ & Proportional Gain & 0.017 \\
\hline$K_{i}$ & Integral Gain & 0.000534 \\
\hline$K_{d}$ & Derivative Gain & 0.00002 \\
\hline
\end{tabular}

and root-mean-square error (RMSE) are calculated using the following equations

$$
\begin{aligned}
& M A X E \% \\
& =\frac{\max (|e(k T)|)}{\max \left(x_{d}(k T)\right)-\min \left(x_{d}(k T)\right)} \times 100 \%
\end{aligned}
$$

\section{RMSE\%}

$$
=\frac{\sqrt{\frac{1}{N} \sum_{k=1}^{N} e^{2}(k T)}}{\max \left(x_{d}(k T)\right)-\min \left(x_{d}(k T)\right)} \times 100 \%
$$

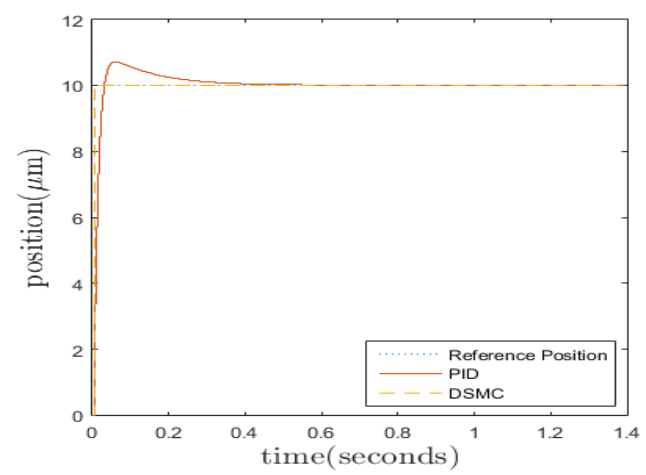

Fig. 5: Tracking performance for step response.

The simulations are performed using MATLAB. In Fig. 5, the reference position is a constant, and PID controller and DSMC are employed with the parameters in Tabs. 2 and 3 . The tracking performance using PID controller show an overshoot of approximately of $1 \mu \mathrm{m}$ and

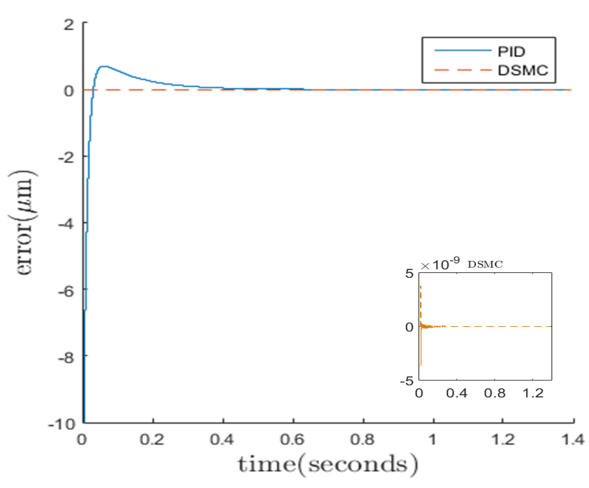

Fig. 6: Error for step response.

Table 3. Parameters of the discrete sliding mode controller

\begin{tabular}{|c|c|c|}
\hline Symbol & Parameter & Value \\
\hline$\lambda_{p}$ & Proportional Gain & 300 \\
\hline$\lambda_{i}$ & Integral Gain & 1 \\
\hline$\lambda_{s}$ & Constant Control Gain & 10000 \\
\hline$\varepsilon$ & Boundary layer & 100 \\
\hline
\end{tabular}

a larger steady state time. However, using a DSMC, the system has a very fast response and an error near to zero as shown in Fig. 6. In addition, the calculate MAXE and RMSE are $3.7904 \times 10^{-10}$ and $1.5346 \times 10^{-12}$ in Tab. 4, respectively.

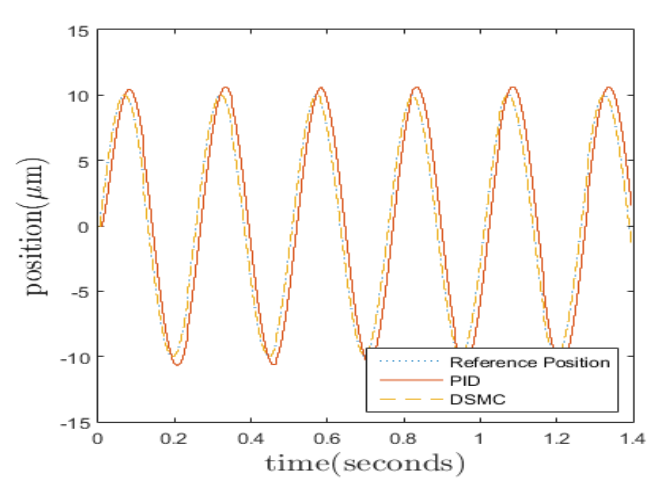

Fig. 7: Tracking performance for sine function.

In the second experiment, the constant value is replaced by a sinusoidal function and the tracking performance is shown in Fig. 7. The tracking performance applying the conventional PID controller has an unsatisfactory tracking if compared to DSMC. 


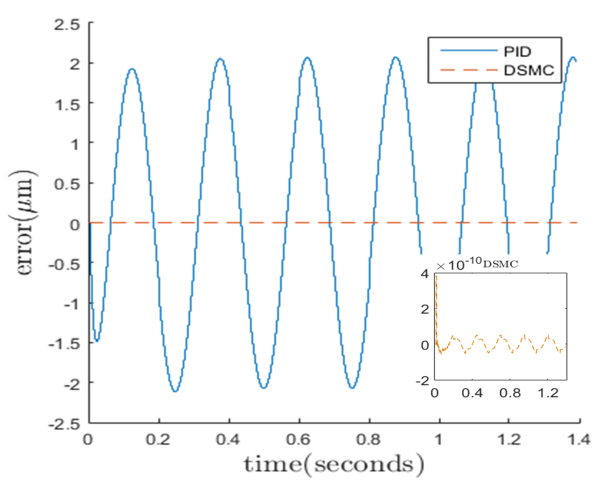

Fig. 8: Error for sine function.

Table 4. MAXE and RMSE

\begin{tabular}{|c|c|c|}
\hline PID & MAXE\% & RMSE\% \\
\hline Constant & 7.05 & 0.26 \\
\hline Sinusoid & 10.35 & 0.39 \\
\hline \multicolumn{3}{|c|}{} \\
\hline DSMC & MAXE $\%$ & RMSE $\%$ \\
\hline Constant & $3.7904 \times 10^{-8}$ & $1.5346 \times 10^{-10}$ \\
\hline Sinusoid & $1.9112 \times 10^{-9}$ & $9.4985 \times 10^{-12}$ \\
\hline
\end{tabular}

From the Tab. 4, this can be explained by the presence of hysteresis effect that turns the system into a complex nonlinear system. For both experiments, DSMC obtains a better trajectory tracking response, tracking errors, MAXE and RMSE in Fig. 7 and Fig. 8 and Tab 4. Similar to the previous simulation, the tracking performance using the proposed DSMC shows no visible overshoot. This improvement is due the combination between disturbance estimation and DSMC which can work well with disturbances and nonlinear plant, resulting in a highperformance micromanipulator.

\section{CONCLUSION}

Trajectory tracking of the piezoelectric bimorph actuator is performed using DSMC in combination with perturbation estimation technique has been simulated in this paper. To describe the hysteresis effect, the Preisach model is adopted and included in the perturbation term. In addition, this simulation was performed without using state observer, using only the position state. This combination attenuates the hysteresis effect have on the system, resulting in a high tracking performance in comparison with the traditional PID controller.

\section{References}

[1] Pota, H.R., Petersen, I.R. and Rana, M.S., 2013. Creep, hysteresis, and cross-coupling reduction in the high-precision positioning of the piezoelectric scanner stage of an atomic force microscope. IEEE Transactions on Nanotechnology, 12(6), pp.11251134.

[2] Zhao, J., Gong, W., Cai, W. and Shang, G., 2013. Piezoelectric bimorph-based scanner in the tip-scan mode for high speed atomic force microscope. Review of Scientific Instruments, 84(8), p.083706.

[3] Evans, J. and Chapman, S., 2014. Characterizing absolute piezoelectric microelectromechanical system displacement using an atomic force microscope. Journal of Applied Physics, 116(6), p.066807.

[4] Bazaei, A., Yong, Y.K., Moheimani, S.R. and Sebastian, A., 2011. Tracking of triangular references using signal transformation for control of a novel AFM scanner stage. IEEE Transactions on Control Systems Technology, 20(2), pp.453-464.

[5] Yong, Y.K., Bhikkaji, B. and Moheimani, S.R.R., 2012. Design, modeling, and FPAA-based control of a high-speed atomic force microscope nanopositioner. IEEE/ASME Transactions on Mechatronics, 18(3), pp.1060-1071.

[6] Acosta, J.C., Polesel-Maris, J., Thoyer, F., Xie, H., Haliyo, S. and Régnier, S., 2013. Gentle and fast atomic force microscopy with a piezoelectric scanning probe for nanorobotics applications. Nanotechnology, 24(6), p.065502.

[7] Gu, G.Y., Zhu, L.M., Su, C.Y. and Ding, H., 2012. Motion control of piezoelectric positioning stages: modeling, controller design, and experimental evaluation. 
IEEE/ASME Transactions on Mechatronics, 18(5), pp.1459-1471.

[8] Esbrook, A., Tan, X. and Khalil, H.K., 2012. Control of systems with hysteresis via servocompensation and its application to nanopositioning. IEEE Transactions on Control Systems Technology, 21(3), pp.725738.

[9] Kenton, B.J. and Leang, K.K., 2011. Design and control of a three-axis serialkinematic high-bandwidth nanopositioner. IEEE/ASME Transactions on Mechatronics, 17(2), pp.356-369.

[10] Xu, Q., 2013. Precision position/force interaction control of a piezoelectric multimorph microgripper for microassembly. IEEE Transactions on Automation Science and Engineering, 10(3), pp.503-514.

[11] Wang, D.H., Yang, Q. and Dong, H.M., 2011. A monolithic compliant piezoelectricdriven microgripper: Design, modeling, and testing. IEEE/ASmE Transactions on mechatronics, 18(1), pp.138-147.

[12] Tamadazte, B., Paindavoine, M., Agnus, J., Pétrini, V. and Piat, N.L.F., 2012. Four dof piezoelectric microgripper equipped with a smart CMOS camera. Journal of Microelectromechanical Systems, 21(2), pp.256-258.

[13] Grossard, M., Boukallel, M., Chaillet, N. and Rotinat-Libersa, C., 2010. Modeling and robust control strategy for a control-optimized piezoelectric microgripper. IEEE/ASME Transactions On Mechatronics, 16(4), pp.674-683.

[14] Rakotondrabe, M. and Ivan, I.A., 2011. Development and force/position control of a new hybrid thermo-piezoelectric microgripper dedicated to micromanipulation tasks. IEEE Transactions on Automation Science and Engineering, 8(4), pp.824-834.

[15] Adriaens, H.J.M.T.S., De Koning, W.L. and Banning, R., 2000. Modeling piezoelectric actuators. IEEE/ASME transactions on mechatronics, 5(4), pp.331-341.
[16] Goldfarb, M. and Celanovic, N., 1997. Modeling piezoelectric stack actuators for control of micromanipulation. IEEE Control Systems Magazine, 17(3), pp.69-79.

[17] Ge, P. and Jouaneh, M., 1997. Generalized Preisach model for hysteresis nonlinearity of piezoceramic actuators. Precision engineering, 20(2), pp.99-111.

[18] Liu, Y., Shan, J., Gabbert, U. and Qi, N., 2013. Hysteresis and creep modeling and compensation for a piezoelectric actuator using a fractional-order Maxwell resistive capacitor approach. Smart Materials and Structures, 22(11), p.115020.

[19] Al Janaideh, M., Rakheja, S. and Su, C.Y., 2009. A generalized Prandtl-Ishlinskii model for characterizing the hysteresis and saturation nonlinearities of smart actuators. Smart Materials and Structures, 18(4), p.045001.

[20] Naser, M., Fuad, M. and Ikhouane, F., 2013. Consistency of the Duhem model with hysteresis. Mathematical Problems in Engineering, 2013.

[21] Ikhouane, F., Hurtado, J.E. and Rodellar, J., 2007. Variation of the hysteresis loop with the Bouc-Wen model parameters. Nonlinear Dynamics, 48(4), pp.361-380.

[22] Saito, T. and Suzuki, T., 1991, June. Reality of chaos in four-dimensional hysteretic circuits. In 1991., IEEE International Sympoisum on Circuits and Systems (pp. 28892892). IEEE.

[23] Fortuna, L., Frasca, M., Graziani, S. and Reddiconto, S., 2006. A chaotic circuit with ferroelectric nonlinearity. Nonlinear Dynamics, 44(1-4), pp.55-61.

[24] Shieh, H.J. and Hsu, C.H., 2008. An adaptive approximator-based backstepping control approach for piezoactuator-driven stages. IEEE Transactions on Industrial Electronics, 55(4), pp.1729-1738.

[25] Bashash, S. and Jalili, N., 2009. Robust adaptive control of coupled parallel piezo-flexural nanopositioning stages. 
IEEE/ASME Transactions on mechatronics, 14(1), pp.11-20.

[26] Khadraoui, S., Rakotondrabe, M. and Lutz, P., 2011. Interval modeling and robust control of piezoelectric microactuators. IEEE Transactions on Control Systems Technology, 20(2), pp.486-494.

[27] Huang, D., Xu, J.X., Venkataramanan, V. and Huynh, T.C.T., 2013. Highperformance tracking of piezoelectric positioning stage using current-cycle iterative learning control with gain scheduling. IEEE Transactions on Industrial Electronics, 61(2), pp.1085-1098.

[28] Young, K.D., Utkin, V.I. and Ozguner, U., 1999. A control engineer's guide to sliding mode control. IEEE transactions on control systems technology, 7(3), pp.328-342.

[29] Elmali, H. and Olgac, N., 1996. Implementation of sliding mode control with perturbation estimation (SMCPE). IEEE transactions on control systems technology, 4(1), pp.79-85.

[30] Xu, Q., 2013. Enhanced discrete-time sliding mode strategy with application to piezoelectric actuator control. IET Control Theory \& Applications, 7(18), pp.2153-2163.

[31] Yan, M. and Shi, Y., 2008. Robust discretetime sliding mode control for uncertain systems with time-varying state delay. IET Control Theory \& Applications, 2(8), pp.662-674.

[32] Lin, C.F., Su, W.C. and Liu, K.H., 2009. Post-filtering approach to output feedback variable structure control for singleinput-single-output sampled-data systems. IET control theory \& applications, 3(8), pp.1145-1154.

[33] Niu, Y., Ho, D.W. and Wang, Z., 2010. Improved sliding mode control for discretetime systems via reaching law. IET control theory \& applications, 4(11), pp.2245-2251.

[34] Abidi, K., Xu, J.X. and Xinghuo, Y., 2007. On the discrete-time integral sliding-mode control. IEEE Transactions on Automatic Control, 52(4), pp.709-715.
[35] Xi, Z. and Hesketh, T., 2010. Discrete time integral sliding mode control for overhead crane with uncertainties. IET control theory \& applications, 4(10), pp.2071-2081.

[36] Xu, Q. and Li, Y., 2011. Model predictive discrete-time sliding mode control of a nanopositioning piezostage without modeling hysteresis. IEEE Transactions on Control Systems Technology, 20(4), pp.983-994.

[37] Xu, J.X. and Abidi, K., 2008. Discretetime output integral sliding-mode control for a piezomotor-driven linear motion stage. IEEE Transactions on Industrial Electronics, 55(11), pp.3917-3926.

[38] Xu, Q. and Li, Y., 2011. Micro/nanopositioning using model predictive output integral discrete sliding mode control. IEEE Transactions on Industrial Electronics, 59(2), pp.1161-1170.

[39] Sha, D. and Bajic, V.B., 2000. Robust discrete adaptive input-output-based sliding mode controller. International Journal of Systems Science, 31(12), pp.1601-1614.

[40] Adriaens, H.J.M.T.S., De Koning, W.L. and Banning, R., 2000. Modeling piezoelectric actuators. IEEE/ASME transactions on mechatronics, 5(4), pp.331-341.

[41] Goldfarb, M. and Celanovic, N., 1997. Modeling piezoelectric stack actuators for control of micromanipulation. IEEE Control Systems Magazine, 17(3), pp.69-79.

[42] Banning, R., De Koning, W.L., Adriaens, H.J. and Koops, R.K., 2001. Statespace analysis and identification for a class of hysteretic systems. Automatica, 37(12), pp.1883-1892.

[43] Coleman, B.D. and Hodgdon, M.L., 1986. A constitutive relation for rate-independent hysteresis in ferromagnetically soft materials. International Journal of Engineering Science, 24(6), pp.897-919. 


\section{About Authors}

Van VAN-HUYNH has completed the Ph.D degree in automation and control from Da-Yeh University, Taiwan. He is currently a Lecturer in the Faculty of Electrical and Electronics Engineering, Ton Duc Thang University, Ho Chi Minh City, Vietnam. He has published totally 10 journal papers and more than 11 international conference papers. His current research interests are in sliding mode control, variable structure control, and power system control.
Phong TRAN-THANH received the B.Eng. degree in physics and electronics with a major in automation and control from the Ho Chi Minh University of Science, Ho Chi Minh, Vietnam, in 2010, and the M.Eng. degree in electrical engineering with a major in automation and control from International University, Ho Chi Minh National University, in 2015. My current research activities include power systems stability and control, time-delay systems, siding mode control, fuzzy and neuron network, dynamic system and application of functional observers to load frequency control of interconnected power systems. 